\title{
Distributed Opportunistic Scheduling: A Control Theoretic Approach
}

\author{
Andres Garcia-Saavedra*, Albert Banchs* ${ }^{* \dagger}$, Pablo Serrano* and Joerg Widmer ${ }^{\dagger}$ \\ ${ }^{*}$ University Carlos III, Madrid, Spain \\ $\dagger$ Institute IMDEA Networks, Madrid, Spain
}

\begin{abstract}
Distributed Opportunistic Scheduling (DOS) techniques have been recently proposed to improve the throughput performance of wireless networks. With DOS, each station contends for the channel with a certain access probability. If a contention is successful, the station measures the channel conditions and transmits in case the channel quality is above a certain threshold. Otherwise, the station does not use the transmission opportunity, allowing all stations to recontend. A key challenge with DOS is to design a distributed algorithm that optimally adjusts the access probability and the threshold of each station. To address this challenge, in this paper we first compute the configuration of these two parameters that jointly optimizes throughput performance in terms of proportional fairness. Then, we propose an adaptive algorithm based on control theory that converges to the desired point of operation. Finally, we conduct a control theoretic analysis of the algorithm to find a setting for its parameters that provides a good tradeoff between stability and speed of convergence. Simulation results validate the design of the proposed algorithm and confirm its advantages over previous proposals.
\end{abstract}

\section{INTRODUCTION}

Communication over wireless channels faces two main challenges inherent to the medium: interference and fading. While the former has traditionally been tackled at the MAC layer (for example through techniques such as CSMA/CA and RTS/CTS), the latter has largely been considered a physical layer problem (and is usually addressed through proper selection of the transmit rate, i.e., channel coding and modulation scheme). However, the physical layer does not always hide fading effects from the MAC layer [1] and using very conservative channel coding and modulation schemes that may allow decoding during deep fades wastes capacity. In contrast, opportunistic scheduling (e.g., [2], [3]) addresses the issue of channel quality variations by preferentially scheduling transmissions of senders with good instantaneous channel conditions. Exploiting knowledge of the channel conditions in this manner has been shown to lead to substantial performance gains.

While centralized opportunistic scheduling mechanisms [2], [3] rely on a central entity with global knowledge of the radio conditions of all stations, the more recent Distributed Opportunistic Scheduling (DOS) techniques [4]-[7], also work in settings where either such a central entity is not available, or the communication overhead to provide timely updates of the channel conditions of all the stations to the central entity is prohibitive.

DOS lets stations contend for channel access and, upon successful contention, a station uses its local information about channel conditions to decide whether to transmit data or give up the transmission opportunity. This decision is taken based on a pure threshold policy, i.e., a station gives up its transmission opportunity if the transmit rate allowed by the channel conditions falls below a certain threshold. By giving up a transmission opportunity and allowing recontention, it is likely that the channel is taken by a station with better channel conditions, resulting in a higher aggregate throughput. Furthermore, since no coordination between stations is required, DOS protocols are simpler to implement and have a lower control overhead compared to centralized opportunistic scheduling mechanisms.

The contributions of [4]-[7] have provided valuable insights and a deeper understanding of DOS techniques and their performance, but less attention has been paid to the design of the algorithms implementing DOS. In our work of [8] we have addressed the issue of optimally configuring DOS; however, the focus of that work is on providing protection against selfish stations. In this paper we propose a novel DOS algorithm that outperforms the previous approaches of [4]-[7] and at the same time continues to perform well in scenarios with non-saturated stations ${ }^{1}$, while relying on less complex mechanisms than [8]. In the following, we review the main contributions of this paper.

The first contribution is the optimal configuration of the DOS parameters. While [4]-[7] only optimize the transmission rate thresholds, we perform a joint optimization of both the thresholds and the access probabilities. Our optimization provides a proportionally fair allocation [9] that achieves a good tradeoff between total throughput and fairness, while [4][7] maximize the total throughput of the network, which may lead to starvation of the stations with poor channel conditions.

The second contribution is a simple adaptive algorithm, based on control theory, that drives the system to the optimal point of operation above. A key advantage of the algorithm over previous proposals [4]-[7] is that it performs well in wireless networks with non-saturated stations. The analysis and design of previous approaches requires the assumption that all stations are always saturated, resulting in overly conservative behavior under non-saturation conditions. In contrast, our approach adapts to the actual network load instead of the

\footnotetext{
${ }^{1}$ A saturated station always has data ready for transmission while a nonsaturated station may at times have nothing to send.
} 
number of stations, and hence naturally lends itself to nonsaturated conditions.

The third contribution of the paper is the control theoretic analysis of the proposed algorithm. This analysis guarantees the convergence and stability of the algorithm, and provides a configuration of its parameters that achieves a good tradeoff between stability and speed of convergence. Prior approaches [4]-[7] do not provide these guarantees.

The rest of the paper is organized as follows. In Section II we present an analysis of our DOS system and, based on this analysis, derive the configuration that optimizes performance in terms of proportional fairness. In Section III we propose a novel adaptive algorithm, named Adaptive Distributed Opportunistic Scheduling (ADOS) that drives the system to the configuration obtained in the previous section. ADOS is analyzed in Section IV from a control theoretic standpoint to derive a configuration of the algorithm's parameters that provides a good tradeoff between stability and reaction to changes. Its performance is validated via simulation in Section V. Finally, Section VI concludes the paper with some final remarks.

\section{DOS Optimal CONFIGURATION}

In the following, we compute the optimal configuration of the access probabilities and transmission rate thresholds in a DOS system for a proportionally fair throughput allocation, which is well-known to provide a good tradeoff between total throughput and fairness [9]. While the analysis conducted in this section assumes saturation conditions, the algorithm that we devise in the next section also takes into account the nonsaturated case.

Our system model follows that of [4]-[7]. We consider a single-hop wireless network with $N$ stations, where time is slotted in mini slots and station $i$ contends for the channel with an access probability $p_{i}$. We assume a collision model where a mini slot contains a collision when two or more stations contend, it contains a successful contention when only one station contends and it is empty otherwise. We let $\tau$ denote the duration of a mini slot. As in [4]-[7], we assume that a station $i$ obtains its local channel conditions upon a successful contention.

After an empty mini slot or a collision, stations recontend for channel access in the next mini slot. Following a successful contention of station $i$, the station may transmit depending on the channel conditions. Let $R_{i}(\theta)$ denote the channel transmission rate of station $i$ at time $\theta$. If $R_{i}(\theta)$ is small (indicating a poor channel), station $i$ gives up on this transmission opportunity and lets all the stations recontend in the next minislot. Otherwise, it transmits after the minislot containing the successful contention for a duration of $\mathcal{T}$.

Our model, like that of [4]-[7], assumes that $R_{i}(\theta)$ remains constant for the duration of a data transmission and that different observations of $R_{i}(\theta)$ are independent. ${ }^{2}$ From [4],

\footnotetext{
${ }^{2}$ The assumption that $R_{i}(\theta)$ remains constant during a data transmission is a standard assumption for the block-fading channel in wireless communications [10], while the assumption that different observations are independent is justified in [4] through numerical calculations.
}

we have that the optimal transmission policy is a threshold policy: given a threshold $\bar{R}_{i}$, station $i$ only transmits after a successful contention if $R_{i}(\theta) \geq \bar{R}_{i}$.

With the above model, stations throughputs are a function of the access probabilities, $\mathbf{p}=\left\{p_{1}, \ldots, p_{N}\right\}$, and the transmission rate thresholds, $\overline{\mathbf{R}}=\left\{\bar{R}_{1}, \ldots, \bar{R}_{N}\right\}$. In the following, we obtain the optimal configuration of these parameters to provide proportional fairness. The analysis to compute these parameters follows that of [8], but it relies on different approximations.

\section{A. Optimal $p_{i}$ configuration}

We start by computing the optimal configuration of the $p_{i}$ parameters. To compute the optimal $p_{i}$ configuration, we start by expressing the throughput $r_{i}$ as a function of $\mathbf{p}$. Let $l_{i}$ be the average number of bits that station $i$ transmits upon a successful contention and $T_{i}$ be the average time it holds the channel. Then, the throughput of station $i$ is

$$
r_{i}=\frac{p_{s, i} l_{i}}{\sum_{j} p_{s, j} T_{j}+\left(1-p_{s}\right) \tau}
$$

where $p_{s, i}$ is the probability that a mini slot contains a successful contention of station $i$

$$
p_{s, i}=p_{i} \prod_{j \neq i}\left(1-p_{j}\right)
$$

and $p_{s}$ is the probability that it contains any successful contention

$$
p_{s}=\sum_{i} p_{s, i}
$$

Both $l_{i}$ and $T_{i}$ depend on $\bar{R}_{i}$. Upon a successful contention, a station holds the channel for a time $\mathcal{T}+\tau$ in case it transmits data and $\tau$ in case it gives up the transmission opportunity. In case the station uses the transmission opportunity, it transmits a number of bits given by $R_{i}(\theta) \mathcal{T}$. Thus, $T_{i}$ and $l_{i}$ can be computed as

$$
T_{i}=\operatorname{Prob}\left(R_{i}(\theta)<\bar{R}_{i}\right) \tau+\operatorname{Prob}\left(R_{i}(\theta) \geq \bar{R}_{i}\right)(\mathcal{T}+\tau)
$$

and

$$
l_{i}=\int_{\bar{R}_{i}}^{\infty} r \mathcal{T} f_{R_{i}}(r) d r
$$

where $f_{R_{i}}(r)$ is the pdf of $R_{i}(\theta)$.

Let us define $w_{i}$ as

$$
w_{i}=\frac{p_{s, i}}{p_{s, 1}}
$$

where we take station 1 as reference. From the above equation we have that $p_{s, i}=w_{i} p_{s} / \sum_{j} w_{j}$ and substituting this in (1) yields

$$
r_{i}=\frac{w_{i} p_{s} l_{i}}{\sum_{j} w_{j} p_{s} T_{j}+\sum_{j} w_{j}\left(1-p_{s}\right) \tau}
$$

In a slotted wireless system such as the one of this paper, the optimal access probabilities satisfy $\sum_{i} p_{i}=1$ (see [11]), 
which gives an optimal success probability $p_{s}$ approximately equal to $1 / e$,

$$
p_{s}=\sum_{i} p_{i} \prod_{j \neq i} 1-p_{j} \approx \sum_{i} p_{i} e^{-\sum_{i} p_{i}}=e^{-1}
$$

With the above, the problem of finding the $\mathbf{p}$ configuration that maximizes the proportionally fair rate allocation is thus equivalent to finding the $w_{i}$ values that maximize $\sum_{i} \log \left(r_{i}\right)$, given that $p_{s}=1 / e$. To obtain these $w_{i}$ values, we impose

$$
\frac{\partial \sum_{i} \log \left(r_{i}\right)}{\partial w_{i}}=0
$$

which yields

$$
\frac{1}{w_{i}}-N \frac{p_{s} T_{i}+\left(1-p_{s}\right) \tau}{\sum_{i} w_{i} p_{s} T_{i}+\sum_{j} w_{j}\left(1-p_{s}\right) \tau}=0
$$

Combining this expression for $w_{i}$ and $w_{j}$, we obtain

$$
\frac{w_{i}}{w_{j}}=\frac{p_{s} T_{j}+\left(1-p_{s}\right) \tau}{p_{s} T_{i}+\left(1-p_{s}\right) \tau}
$$

Given that $w_{i} / w_{j} \approx p_{i} / p_{j}$, the above can be rewritten as

$$
\frac{p_{i}}{p_{j}}=\frac{T_{j}+(e-1) \tau}{T_{i}+(e-1) \tau}
$$

Furthermore, the probability that a given mini slot is empty can be computed as follows,

$$
p_{e}=\prod_{i} 1-p_{i} \approx e^{-\sum_{i} p_{i}}=e^{-1}
$$

With the above, we compute the solution of the optimization problem by finding the $\mathbf{p}$ values that solve the system of equations formed by (12) and (13). The uniqueness of the solution of this system of equations can be proved as follows. Without loss of generality, let us take the access probability of station $1, p_{1}$, as reference. From (12) we have that $p_{i}$ for $i \neq 1$ can be expressed as a continuous and monotone increasing function of $p_{1}$. Applying this to (13), we have that the term $\left(\prod_{i} 1-p_{i}\right)$ is a continuous and monotone decreasing function of $p_{1}$ that starts at 1 and decreases to 0 , while the right hand side is a constant value $0<1 / e<1$. From this, it follows that there is a unique value of $p_{1}$ that satisfies this equation. Taking the resulting $p_{1}$ and computing $p_{i} \forall i \neq 1$ from (12), we have a solution to the system. Uniqueness of the solution is given by the fact that all relationships are bijective and any solution must satisfy (13), which (as we have shown) has only one solution.

Hereafter we denote the unique solution to the system of equations by $\mathbf{p}^{*}=\left\{p_{1}^{*}, \ldots, p_{N}^{*}\right\}$. Note that determining $\mathbf{p}^{*}$ requires computing $T_{i} \forall i$, which depend on the optimal configuration of the thresholds $\overline{\mathbf{R}}$. In the following section we address the computation of the optimal $\overline{\mathbf{R}}$, which we denote by $\overline{\mathbf{R}}^{*}=\left\{\bar{R}_{1}^{*}, \ldots, \bar{R}_{N}^{*}\right\}$.

\section{B. Optimal $\bar{R}_{i}$ configuration}

In order to obtain the optimal configuration of $\overline{\mathbf{R}}$, we need to find the transmission threshold of each station that, given the $\mathbf{p}^{*}$ computed above, optimizes the overall performance in terms of proportional fairness.

In the following we show that the optimal configuration of the transmission thresholds is given by $\bar{R}_{k}^{*}=\bar{R}_{k}^{1}$, where $\bar{R}_{k}^{1}$ is the transmission rate threshold that optimizes the throughput of station $k$ when it is alone in the channel and contends with $p_{k}=1 / e$ (under the assumption that different channel observations are independent).

We prove that $\bar{R}_{k}^{1}$ optimizes performance by contradiction: we assume that there exists a configuration $\overline{\mathbf{R}}^{*}$ with $\bar{R}_{k}^{*} \neq \bar{R}_{k}^{1}$ for some station $k$ that provides proportional fairness, and show that, if this were the case, we could find an alternative configuration that provides a larger $\sum_{i} \log \left(r_{i}\right)$, which contradicts the initial assumption.

Let $l_{k}^{1}$ and $T_{k}^{1}$ be the values of $l_{k}$ and $T_{k}$ for the threshold $\bar{R}_{k}^{1}$ and $l_{k}^{*}$ and $T_{k}^{*}$ the corresponding values for $\bar{R}_{k}^{*}$. Since $\bar{R}_{k}^{1}$ maximizes $r_{k}$ when station $k$ is alone:

$$
\frac{l_{k}^{1}}{T_{k}^{1}+(e-1) \tau}>\frac{l_{k}^{*}}{T_{k}^{*}+(e-1) \tau}
$$

Let us consider that there are $N$ stations in the network and the configuration $\overline{\mathbf{R}}^{*}$ is used. Given $\overline{\mathbf{R}}^{*}$, the $\mathbf{p}^{*}$ that maximizes $\sum_{i} \log \left(r_{i}\right)$ is given by (12) and (13). This leads to the following throughput for station $k$ :

$$
r_{k}^{*}=\frac{p_{s, k}^{*} l_{k}^{*}}{\sum_{j} p_{s, j}^{*}\left(T_{j}^{*}+(e-1) \tau\right)}=\frac{l_{k}^{*}}{N\left(T_{k}^{*}+(e-1) \tau\right)}
$$

and for the other stations:

$$
r_{i}^{*}=\frac{l_{i}^{*}}{N\left(T_{i}^{*}+(e-1) \tau\right)}, \forall i \neq k
$$

Let us now consider the alternative configuration $\bar{R}_{k}^{1}$ for station $k$ and $\bar{R}_{i}^{*}$ for the other stations. Let us take the $p_{k}^{1}$ and $p_{i}^{1}$ configuration that satisfies (12) and (13) with this alternative configuration. This yields the following throughput for station $k$ :

$$
r_{k}^{1}=\frac{l_{k}^{1}}{N\left(T_{k}^{1}+(e-1) \tau\right)}>r_{k}^{*}
$$

and for the other stations:

$$
r_{i}^{*}=\frac{l_{i}^{*}}{N\left(T_{i}^{*}+(e-1) \tau\right)}, \forall i \neq k
$$

With the above, we have found an alternative configuration that provides a higher throughput to station $k$ and the same throughput to all other stations. Therefore, this alternative configuration increases $\sum_{i} \log \left(r_{i}\right)$, which contradicts the initial assumption that the configuration $\overline{\mathbf{R}}^{*}$ provides proportional fairness.

Following the above result, the optimal configuration of the threshold $\bar{R}_{i}^{*}$ can be obtained by computing the transmission rate threshold $\bar{R}_{k}^{1}$ that optimizes the throughput of station $k$ when it is alone in the channel and it contends for the channel 
with $p_{k}=1 / e$. This is done in [4], which uses optimal stopping theory and finds that the optimal threshold can be obtained by solving the following fixed point equation:

$$
E\left[R_{i}(\theta)-\bar{R}_{i}^{*}\right]^{+}=\frac{\bar{R}_{i}^{*} \tau}{\mathcal{T} / e}
$$

Note that the above allows computing the threshold $\bar{R}_{i}^{*}$ of a station based on local information only, as the equation does not depend on the other stations in the network and their radio conditions.

\section{ADOS ALGORITHM}

In this section we propose the ADOS mechanism that aims at driving the system to the optimal point of operation both under saturation and under non-saturation conditions. The optimal configuration $\left\{\mathbf{p}^{*}, \overline{\mathbf{R}}^{*}\right\}$ obtained in the previous section corresponds to the case where all stations are saturated. We next discuss how to consider the case when some of the stations are not saturated.

In the previous section we have seen that, when all the stations are saturated, the optimal channel empty probability $p_{e}$ takes a constant value equal to $1 / e$, independent of the number of stations. The first key approximation is to assume that this also holds when some of the stations are not saturated. The rationale behind this assumption is that when some of the stations are not saturated, they transmit with a smaller access probability, and therefore the other stations should transmit with a higher access probability to achieve optimal throughput efficiency, as otherwise channel time is wasted with empty mini slots. By keeping the same target $p_{e}$, we increase the access probabilities of the other stations, and thus adapt their behavior to the actual load in the network.

We have also seen in the previous section that, under saturation, the optimal transmission thresholds are constant values that only depend on the local radio conditions. The second key approximation is to assume that the optimal transmission thresholds take the same constant values under non-saturation. Note that, according to the analysis of the previous section, these thresholds only depend on the time wasted in contention. As we adjust the access probabilities to the actual load, we can assume that the time wasted in contention is the same as in the saturated case, which leads to the same optimal configuration for the thresholds.

Following the above two assumptions, with ADOS each station maintains a fixed $\bar{R}_{i}$ configuration, which is computed from (19) based on local information only, and implements an adaptive algorithm to configure the access probability $p_{i}$, with the goal of driving the channel empty probability to $1 / e$. One of the key features of ADOS is that it neither requires any knowledge about the number of stations in the network nor their channel conditions.

Driving the channel empty probability toward a constant optimum value fits well with the framework of classic control theory. With these techniques, we measure the output signal of the system and, by judiciously adjusting the control signal, we aim at driving it to the reference signal given by a

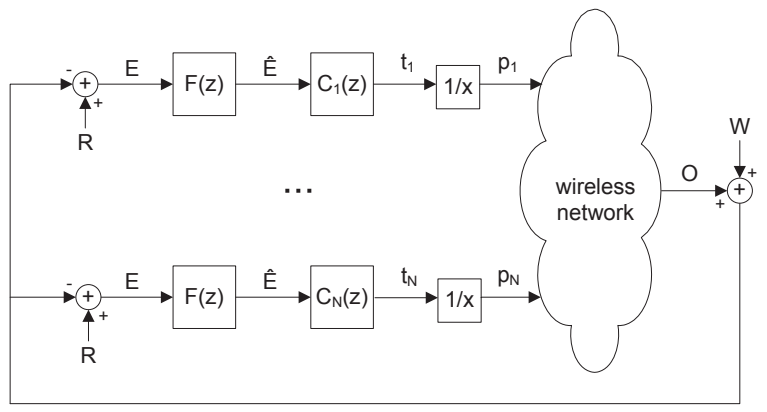

Fig. 1. ADOS system.

constant value. A key advantage of using such techniques is that they provide the means for achieving a good tradeoff between the speed of reaction and stability while guaranteeing convergence, which is a major challenge when designing adaptive algorithms.

Fig. 1 depicts our system, where each station computes the error signal $E$ by subtracting the output signal $O$ from the reference signal $R$. The output signal $O$ is combined with a noise component $W$ of zero mean, modeling the randomness of the channel access mechanism. In order to eliminate this noise, we follow the design guidelines from [12] and introduce a low-pass filter $F(z)$. The filtered error signal $\hat{E}$ is then fed into the controller $C_{i}(z)$ of each station, which provides the control signal $t_{i}$, corresponding to the average time between two transmission of station $i$. Station $i$ then computes its access probability as $p_{i}=1 / t_{i}$. With the $p_{i}$ of each station, the wireless network provides the output signal $O$, which closes the loop.

In the above system, we need to design the reference and output signals $R$ and $O$, as well as the transfer functions of the low-pass filter and the controller, $F(z)$ and $C_{i}(z)$. In the following we address the design of these components with the goal of ensuring that the empty probability $p_{e}$ is driven to $1 / e$.

In our system, time is divided into intervals such that the end of an interval corresponds to a transmission in the channel (either a success or a collision). Given that the target empty probability is equal to $1 / e$, the target average number of empty mini slots between two transmissions (i.e., our reference signal) is equal to $R=1 /(e-1)$. In this way, after the $n$ th transmission, each station computes the output signal at interval $n$, denoted as $O(n)$, as the number of empty mini slots between the $(n-1)$-th and the $n$-th transmission. The error signal for the next interval is then computed as

$$
E(n+1)=R-O(n) .
$$

With the above, if $p_{e}$ is too large then $O(n)$ will be larger than $R$ in average, yielding a negative error signal $E(n+1)$ that will decrease $t_{i}$ for the next interval, which will increase the access probability $p_{i}$ and therefore reduce $p_{e}$ (and viceversa). This ensures that $p_{e}$ will be driven to the optimal value. Note also that our reference signal is a constant independent of the number of stations and their channel conditions, which 


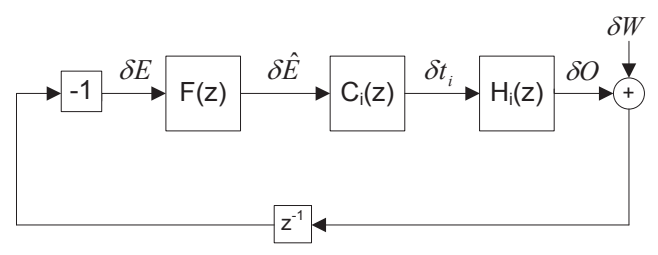

Fig. 2. Closed-loop system for station $i$.

is an essential requirement in the design of control theory systems.

For the low-pass filter $F(z)$, we use a simple exponential smoothing algorithm of parameter $\alpha$ [13],

$$
\hat{E}(n)=\alpha E(n)+(1-\alpha) \hat{E}(n-1)
$$

which corresponds to the following transfer function

$$
F(z)=\frac{\alpha}{1-(1-\alpha) z^{-1}}
$$

For the transfer function of the controllers $C_{i}(z)$, we use a very simple controller from classical control theory, namely the Proportional Controller [14], which has already been used in networking problems (see e.g. [15]):

$$
C_{i}(z)=K_{p, i}
$$

where $K_{p, i}$ is a per-station constant.

In addition to driving the empty probability to $1 / e$, we also impose that the access probabilities satisfy $(12)^{3}$. Since we feed the same error into the different stations, and the proportional controller simply multiplies this error by a constant to compute $p_{i}$, the following equation holds for all $i, j$ :

$$
\frac{p_{i}}{p_{j}}=\frac{K_{p, j}}{K_{p, i}}
$$

Therefore, by simply setting $K_{p, i}$ as

$$
K_{p, i}=K_{p}\left(T_{i}+(e-1) \tau\right)
$$

we ensure that (12) is satisfied.

With the above, we have all the components of the ADOS algorithm fully designed. The remaining challenge is the setting of the parameters $K_{p}$ and $\alpha$. In the following section we conduct a control theoretic analysis of the algorithm to find a suitable parameter setting.

\section{Control Theoretic Analysis}

To find good values for the parameters $K_{p}$ and $\alpha$, we conduct a control theoretic analysis of the closed-loop system for station $i$ depicted in Fig. 2. Note that the term $z^{-1}$ in the figure shows that the error signal $E$ at a given interval is computed with the output signal $O$ of the previous interval.

In order to analyze this system from a control theoretic standpoint, we need to characterize the transfer function $H_{i}$,

\footnotetext{
${ }^{3}$ In Section II we have seen that (12) needs to be satisfied by the saturated stations. For the non-saturated stations, the access probability depends on the sending behavior and not on $p_{i}$. Therefore, we can impose (12) on all stations and thus avoid differentiating saturated from non-saturated stations.
}

which takes $t_{i}$ as input and gives $O$ as output. The following equation gives a nonlinear relationship between $O$ and $\mathbf{t}$ :

$$
O=\frac{1}{1-p_{e}}-1
$$

where

$$
p_{e}=\prod_{j}\left(1-1 / t_{j}\right)
$$

To express the above relationship as a transfer function, we linearize it when the system suffers small perturbations around its stable point of operation. We then study the linearized model and force that it is stable. Note that the stability of the linearized model guarantees that our system is locally stable. ${ }^{4}$

We express the perturbations around the stable point of operation as follows:

$$
t_{i}=t_{i}^{*}+\delta t_{i}
$$

where $t_{i}^{*}=1 / p_{i}^{*}$ is the stable point of operation of $t_{i}$.

With the above, the perturbations suffered by $O$ can be approximated by

$$
\delta O=\sum_{j} \frac{\partial O}{\partial t_{j}} \delta t_{j}
$$

where

$$
\frac{\partial O}{\partial t_{j}}=\frac{\partial O}{\partial p_{j}} \frac{\partial p_{j}}{\partial t_{j}}=\frac{p_{e} p_{j}^{2}}{\left(1-p_{j}\right)\left(1-p_{e}\right)^{2}}
$$

Given that $t_{i} / t_{j}=\left(T_{i}+(e-1) \tau\right) /\left(T_{j}+(e-1) \tau\right)$, the above can be rewritten as

$$
\delta O=\left(\sum_{j} \frac{\left(T_{j}+(e-1) \tau\right) p_{e} p_{j}^{2}}{\left(T_{i}+(e-1) \tau\right)\left(1-p_{j}\right)\left(1-p_{e}\right)^{2}}\right) \delta t_{i}
$$

With the above, we have characterized $H_{i}$ :

$$
H_{i}=\sum_{j} \frac{\left(T_{j}+(e-1) \tau\right) p_{e} p_{j}^{2}}{\left(T_{i}+(e-1) \tau\right)\left(1-p_{j}\right)\left(1-p_{e}\right)^{2}}
$$

The closed-loop transfer function for station $i$ is then given by

$$
T_{i}(z)=\frac{-z^{-1} C_{i}(z) F(z) H_{i}(z)}{1+z^{-1} C_{i}(z) F(z) H_{i}(z)}
$$

Substituting the expressions for $F(z), C_{i}(z)$ and $H_{i}(z)$ yields

$$
T_{i}(z)=\frac{-\alpha H_{i} K_{p, i}}{z-\left(1-\alpha-\alpha K_{p, i} H_{i}\right)}
$$

To guarantee stability, we need to ensure that the zero of the denominator of $T_{i}(z)$ falls inside the unit circle $|z|<1$ [17], which implies

$$
K_{p}<\frac{2-\alpha}{\alpha} \frac{1}{\sum_{j} \frac{\left(T_{j}+(e-1) \tau\right) p_{e} p_{j}^{2}}{\left(1-p_{j}\right)\left(1-p_{e}\right)^{2}}}
$$

The problem with the above upper bound is that it depends on the number of stations and their channel conditions. In

\footnotetext{
${ }^{4} \mathrm{~A}$ similar approach was used in [16] to analyze RED from a control theoretic standpoint.
} 
order to assure stability, we need to obtain an upper bound that guarantees stability independent of these parameters. To do this, we observe that the right hand side of the above inequality takes a minimum value when $N=1$ and $T_{1}=\tau+\mathcal{T}$. Therefore, by setting $K_{p}$ as follows, we guarantee that the above inequality will be met independent of the number of stations and their channel conditions:

$$
K_{p}<\frac{2-\alpha}{\alpha(\mathcal{T}+e \tau)}
$$

The above provides the maximum $K_{p}$ value that guarantees stability, which we denote by $K_{p}^{\max }$,

$$
K_{p}^{\max }=\frac{2-\alpha}{\alpha(\mathcal{T}+e \tau)}
$$

In order to set $K_{p}$ to a value that provides a good tradeoff between the speed of reaction to changes and stability, we follow the Ziegler-Nichols rules [14], which are widely used to configure proportional controllers. According to these rules, this parameter cannot be larger than one half of the maximum value that guarantees stability, which we denote by $K_{p}^{\text {stability }}$

$$
K_{p} \leq K_{p}^{\text {stability }}=\frac{K_{p}^{\max }}{2}
$$

In addition to the above, $K_{p}$ also needs to be set to eliminate the noise from the system. Noise is generated by the randomness of the output signal, which is a geometric random variable of factor $1-p_{e}=1-1 / e$. Hence, the noise at the input of the low-pass filter has a zero mean and a variance given by:

$$
E\left[W^{2}\right]=\frac{p_{e}}{\left(1-p_{e}\right)^{2}}=\frac{1 / e}{(1-1 / e)^{2}}
$$

The noise at the output of the controller can be obtained from the noise at the input of the low-pass filter with the following transfer function:

$$
T_{N}(z)=\frac{-z^{-1} C_{i}(z) F(z)}{1+z^{-1} C_{i}(z) F(z) H_{i}(z)}
$$

Substituting $C_{i}(z), F(z)$ and $H_{i}(z)$ into the above yields

$$
T_{N}(z)=\frac{-z^{-1} \alpha K_{p, i}}{1-z^{-1}\left(1-\alpha\left(1+K_{p, i} H_{i}\right)\right)}
$$

With the above transfer function, we can compute the variance of the noise at the output of the controller, denoted by $W_{C}$, as follows:

$$
E\left[W_{C}^{2}\right]=\frac{\alpha^{2} K_{p, i}^{2}}{1-\left(1-\alpha\left(1+K_{p, i} H_{i}\right)\right)^{2}} E\left[W^{2}\right]
$$

From the above equation, and taking into account from (34) and (38) that $\alpha\left(1+K_{p, i} H_{i}\right) \leq 1+\alpha / 2$, we can obtain the following upper bound for $E\left[W_{C}^{2}\right]$ :

$$
E\left[W_{C}^{2}\right] \leq \frac{\alpha K_{p, i}}{(1-\alpha / 2) H_{i}} E\left[W^{2}\right]
$$

To limit the impact of the noise, we impose a gain factor of at least $G$ of the signal level at the output of the controller, $E\left[S^{2}\right]$, over the noise level at the same point, $E\left[W_{C}^{2}\right]$ :

$$
\frac{E\left[S^{2}\right]}{E\left[W_{C}^{2}\right]} \geq G
$$

The signal at the output of the controller is equal to $t_{i}$, which yields $E\left[S^{2}\right]=t_{i}^{2}$. Combining this with the inequality of (43), we have that the following condition is sufficient to provide the desired gain:

$$
\frac{t_{i}^{2}(1-\alpha / 2) H_{i}}{\alpha K_{p, i} E\left[W^{2}\right]} \geq G
$$

Isolating $K_{p}$ from the above yields

$$
K_{p} \leq \frac{t_{i}^{2}(1-\alpha / 2)}{G \alpha E\left[W^{2}\right]} \sum_{j} \frac{\left(T_{j}+(e-1) \tau\right) p_{e} p_{j}^{2}}{\left(T_{i}+(e-1) \tau\right)^{2}\left(1-p_{j}\right)\left(1-p_{e}\right)^{2}}
$$

which is satisfied as long as the following condition holds,

$$
K_{p} \leq \frac{1-\alpha / 2}{G \alpha} \sum_{j} \frac{T_{j}+(e-1) \tau}{\left(T_{i}+(e-1) \tau\right)^{2}}
$$

To find an upper bound that is independent of the number of stations and their conditions, we observe that the right hand side of the above inequality takes a minimum for $N=1$ and $T_{1}=\tau+\mathcal{T}$, which leads to the following upper bound which we denote by $K_{p}^{\text {noise }}$,

$$
K_{p} \leq K_{p}^{\text {noise }}=\frac{1-\alpha / 2}{G \alpha(\mathcal{T}+e \tau)}
$$

The analysis conducted in this section has given two upper bounds, $K_{p}^{\text {stability }}$ and $K_{p}^{\text {noise }}$, which guarantee that on the one hand the system behaves stably and on the other hand the noise level is not excessive. As these bounds depend on $\alpha$ and $G$, we also need to find a setting for these parameters. We set $G=10^{2}$ and $\alpha=10^{-4}$ to provide a good level of protection against noise while allowing sufficiently large $K_{p, i}$ values, which is needed to avoid a large steady state error at the input of the controllers. With these $\alpha$ and $G$ values, we configure $K_{p}$ as follows:

$$
K_{p}=\min \left(K_{p}^{\text {noise }}, K_{p}^{\text {stability }}\right)
$$

which ensures that the two objectives concerning stability and noise are met.

\section{Performance Evaluation}

In this section we present a performance evaluation of ADOS by means of simulations. Unless otherwise stated, we assume that different observations of the channel conditions are independent and that the available transmission rate for a given SNR is given by the Shannon channel capacity:

$$
R(h)=W \log _{2}\left(1+\rho|h|^{2}\right) \quad \text { bits/s }
$$

where $W$ is the channel bandwidth, $\rho$ is the normalized average SNR and $h$ is the random gain of Rayleigh fading.

We implemented the ADOS algorithm in OMNET++. In the simulations, we set $W=10^{7}$ and $\mathcal{T} / \tau=10$. For all results, $95 \%$ confidence intervals are below $0.5 \%$. 


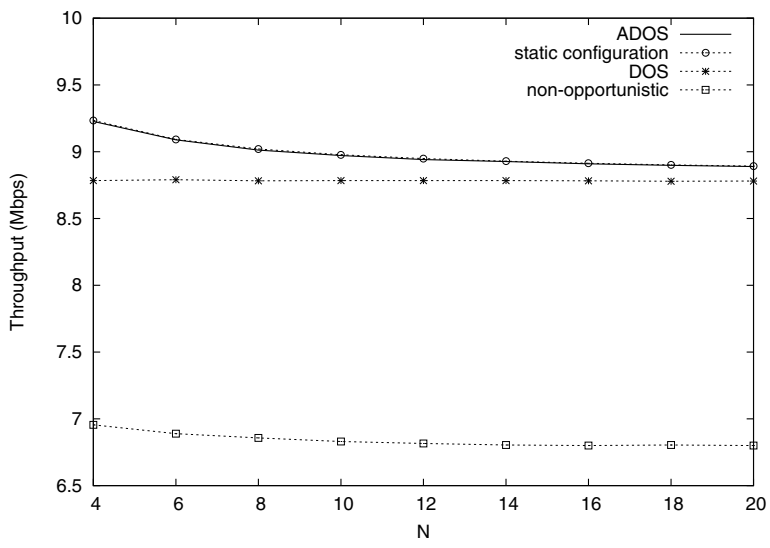

Fig. 3. Homogeneous scenario with saturated stations.

\section{A. Homogeneous scenario with saturated stations}

We start by considering a homogeneous scenario where all stations are saturated and have the same normalized average SNR $\left(\rho_{i}=1 \forall i\right)$. We compare the performance of ADOS to the following approaches: (i) the static optimal configuration obtained from performing an exhaustive search over the $\left\{p_{i}, \bar{R}_{i}\right\}$ space and choosing the best configuration ('static configuration'), (ii) the approach proposed in [4] ('DOS'), and (iii) an approach that does not perform opportunistic scheduling but always transmits after successful contention ('non-opportunistic'). ${ }^{5}$ Fig. 3 shows the total throughput as a function of the number of stations in the network. The figure confirms that ADOS is effective in driving the system to the optimal point of operation, providing the same throughput as the static configuration. The DOS approach provides smaller throughputs as it only optimizes the transmission thresholds, while the non-opportunistic approach provides an even lower throughput due to the lack of opportunistic scheduling. In conclusion, the proposed ADOS algorithm provides optimal throughput performance, outperforming the other approaches.

\section{B. Heterogeneous scenario with saturated stations}

In the case of heterogeneous channel conditions, performance does not only depend on the total throughput but also on the way this throughput is shared among the stations. To analyze performance in this scenario, we consider $N=20$ saturated stations divided into four groups according to their channel conditions. The normalized SNR of the stations from group $i$ is given by $\rho_{i}=1+(i-1) \Delta \rho$, with $i \in\{1,2,3,4\}$. Fig. 4 shows $\sum_{i} \log \left(r_{i}\right)$, the figure of merit for proportional fairness, as a function of $\Delta \rho$. We observe that ADOS performs at the same level as the benchmark given by the static configuration, while the DOS and non-opportunistic approaches provide a substantially lower performance: DOS exhibits an

\footnotetext{
${ }^{5}$ Since [4] only optimizes the transmission thresholds but not the access probabilities, for the 'DOS' approach we take the configuration of access probabilities that are used in the simulation results of [4]. For the "nonopportunistic' approach, we choose the access probabilities that maximize the performance, by adapting the analysis of Section II to the case when stations always transmit after successful contention.
}

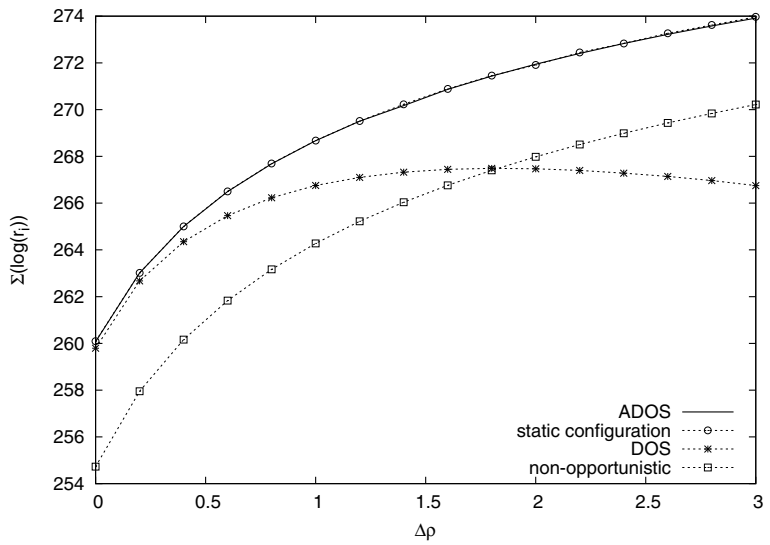

Fig. 4. Heterogeneous scenario with saturated stations.

increasing degree of unfairness as $\Delta \rho$ grows that harms its performance in terms of proportional fairness, while the nonopportunistic approach has lower throughput due to the lack of opportunistic scheduling. In order to gain insight into the degree of unfairness of $D O S$, we compute the throughput performance of a station from group 1 and a station from group 4 , for the case of $\Delta \rho=3$. With ADOS, the throughputs are $r_{1}=1.31 \mathrm{Mbps}$ and $r_{4}=0.44$, while for the case of DOS these throughputs are $r_{1}=1.76 \mathrm{Mbps}$ and $r_{4}=0.06$. These results confirm that $D O S$ suffers from high unfairness with heterogeneous radio conditions.

\section{Homogeneous scenario with non-saturated stations}

The experiments conducted in previous sections considered saturated conditions, i.e., all stations always had data ready for transmission. In order to assess performance in the case of non-saturation, we consider a scenario with homogeneous radio conditions, with one saturated station and $N-1$ stations transmitting at half their saturation throughput (i.e., the throughput the would obtain if they were saturated). Fig. 5 illustrates the total throughput of the wireless network as a function of the number of stations, showing that ADOS significantly outperforms all other approaches. The reason is that the other approaches assume that all stations are always saturated, and therefore the access probabilities they use become overly conservative for the non-saturated case.

\section{Heterogeneous scenario with non-saturated stations}

To evaluate the performance improvement achieved by ADOS with non-saturated stations in the case of an heterogeneous scenario, we repeat the experiment of Fig. 4 with one of the stations with the highest SNR saturated and the rest of the stations sending at half their saturation throughput. The results, given in Fig. 6, show that ADOS also substantially outperforms the other approaches in this case. We further observe that as $\Delta \rho$ grows, the performance of DOS becomes worse than that of the other approaches but does not degrade as in Fig. 4. The reasons for this are twofold. On the one hand, there are no fairness issues as only one station is saturated. On the other hand, as $\Delta \rho$ grows, DOS increases the $R_{i}^{*}$ of all 


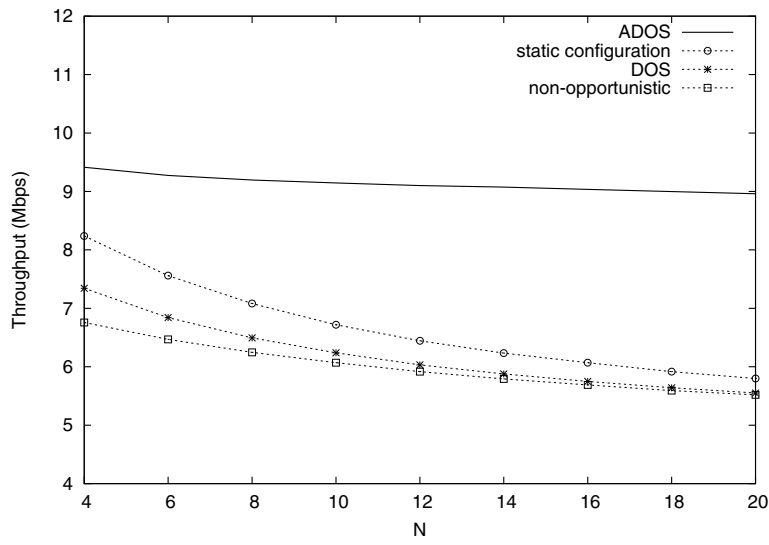

Fig. 5. Homogeneous scenario with non-saturated stations.

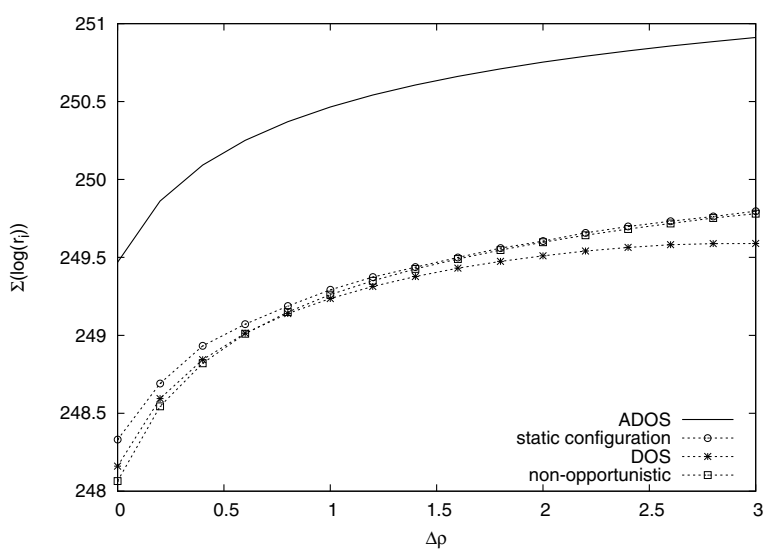

Fig. 6. Heterogeneous scenario with non-saturated stations.

stations and as a result the non-saturated stations with a small SNR need more successful contentions to send their traffic.

\section{E. Impact of channel coherence time}

Our channel model is based on the assumption that different observations of the channel conditions are independent. In order to understand the impact of this assumption, we repeat the experiment of Fig. 5 using Jakes' channel model [18] to obtain channel conditions that are correlated over time. The results, for a Doppler frequency of $f_{D}=2 \pi / 100 \tau$, are given in Fig. 7 . We first observe that ADOS outperforms very substantially all the other approaches, which validates its effectiveness also in this case. We also observe that the throughput obtained is smaller than that of Fig. 5. This is due to the fact that when the channel is bad, a station does not transmit after a successful contention, and therefore it takes a shorter time until it successfully contends again. As a result, a station accesses the channel more often when the channel is bad than when it is good, which introduces a bias that reduces throughput. We finally observe that performance increases with $N$, which is caused by the fact that the larger $N$, the less likely is that a station that gives up a transmission opportunity wins the next contention before its channel conditions improve.

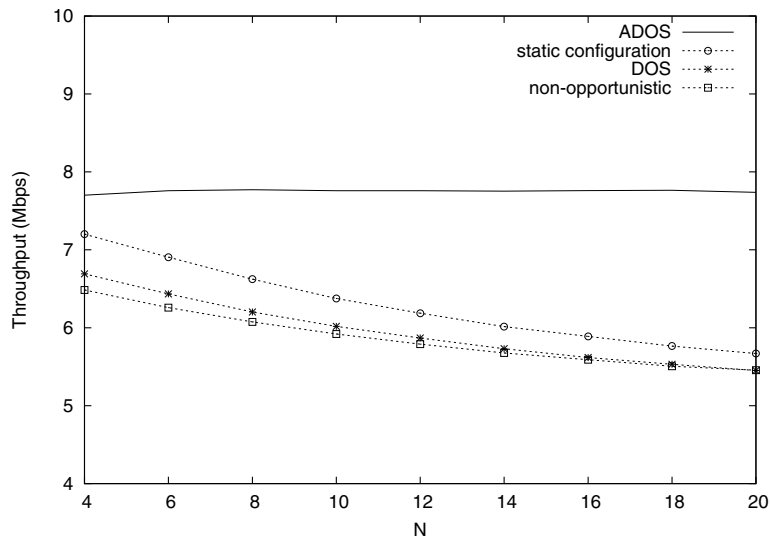

Fig. 7. Performance with Jakes' channel model.

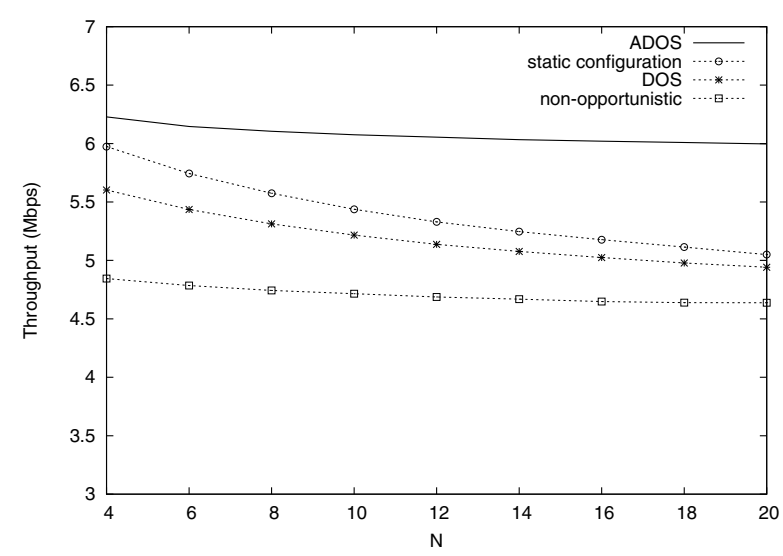

Fig. 8. Throughput performance for a discrete set of rates.

\section{F. Discrete set of transmission rates}

While all previous experiments assumed continuous rates, our analysis as well as the design of ADOS do not rely on any assumption on the mapping of SNR to transmission rates, and therefore any mapping function (continuous or discrete) can be used. To show that ADOS is effective when only a set of discrete rates is allowed, we consider the case of a wireless system in which the only transmission rates available are $\{1,2,5.5,12,24,48,54\}$ Mbps. For a given SNR, we choose the largest available transmission rate that is smaller than the one given by (50). We repeat the experiment of Fig. 5 with discrete rates. The results in Fig. 8 confirm that ADOS outperforms the other approaches, and hence shows that the proposed algorithm also works well in the case of a discrete set of transmission rates. Note that the resulting throughputs are lower, since for a given SNR, a station cannot use the maximum transmission rate supported by this SNR but needs to use a smaller one from the set of available rates.

\section{G. Stability}

The setting of the $K_{p}$ and $\alpha$ parameters proposed in Section IV achieves a good tradeoff between stability and speed of reaction. This is verified by the results presented in this and the following sections. 

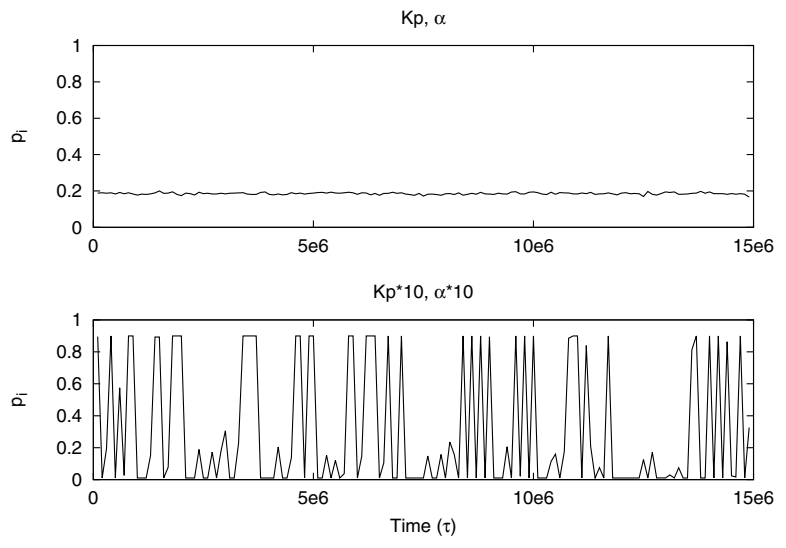

Fig. 9. Stability

To verify stable behavior, we analyze the evolution over time of the access probability $p_{i}$ of a station for the proposed $\left\{K_{p}, \alpha\right\}$ setting and for a configuration of these parameters 10 times larger, in a homogeneous scenario with $N=5$ saturated stations. Fig. 9 shows the evolution of $p_{i}$ for both cases, sampled over $10^{5} \tau$ intervals. We observe from the figure that with the proposed setting (labeled " $K_{p}, \alpha$ "), $p_{i}$ shows minor deviations around its average value, while for a larger setting (labeled " $K_{p} * 10, \alpha * 10$ "), it shows unstable behavior with drastic oscillations.

\section{H. Speed of reaction}

We next investigate the speed with which the system reacts to changes. To this aim, in a wireless network with initially 5 stations, 5 additional stations join the network after a time $5 \cdot 10^{6} \tau$. Fig. 10 shows the evolution of the access probability of one of the initial stations sampled over $10^{5} \tau$ intervals. We observe from the figure that with our setting (labeled " $K_{p}, \alpha$ "), the system quickly adapts the $p_{i}$ of the station to the new value. In contrast, for a setting of these parameters 10 times smaller (labeled " $K_{p} / 10, \alpha / 10$ "), the reaction is very slow and the system only converges after $5 \cdot 10^{6} \tau$.

The results confirm that the proposed configuration provides a good tradeoff between stability and speed of reaction, since with a larger setting of $\left\{K_{p}, \alpha\right\}$ the system suffers from instability, while with a smaller setting it reacts too slowly.

\section{CONCLUSIONS}

Distributed Opportunistic Scheduling (DOS) techniques provide throughput gains in wireless networks without requiring a centralized scheduler. One of the challenges of these techniques is the design of an adaptive algorithm that adjusts the DOS parameters to their optimal value. In this paper we propose a novel algorithm, named ADOS, with the following advantages: $(i)$ it jointly optimizes both the access probabilities and the transmission thresholds; (ii) it performs better than any of the previous approaches, in particular under non-saturation conditions; (iii) it provides a good tradeoff between total throughput and fairness; and (iv) it guarantees convergence and stability.

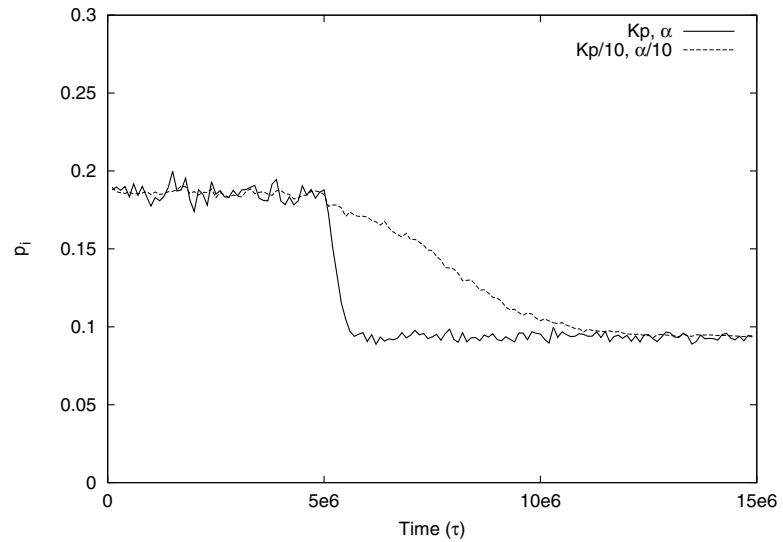

Fig. 10. Speed of reaction

\section{REFERENCES}

[1] M. Cao, V. Raghunathan, and P. Kumar, "Cross-layer exploitation of mac layer diversity in wireless networks," in Proceedings of IEEE ICNP, Santa Barbara, CA, November 2006.

[2] P. Viswanath, D. N. Tse, and R. Laroia, "Opportunistic beamforming using dumb antennas," IEEE Transactions on Information Theory, vol. 48, no. 6, pp. 1277-1294, June 2002.

[3] M. Andrews et al., "Providing quality of service over a shared wireless link," IEEE Communications Magazine, vol. 39, no. 2, February 2001.

[4] D. Zheng, W. Ge, and J. Zhang, "Distributed opportunistic scheduling for ad hoc networks with random access: an optimal stopping approach," IEEE Transactions on Information Theory, vol. 55, no. 1, January 2009.

[5] P. Thejaswi, J. Zhang, M.-O. Pun, H. V. Poor, and D. Zheng, "Distributed opportunistic scheduling with two-level probing," IEEE/ACM Transactions on Networking, vol. 18, no. 5, October 2010.

[6] D. Zheng, , M.-O. Pun, W. Ge, H. V. Poor, and J. Zhang, "Distributed opportunistic scheduling for ad hoc communications with imperfect channel information," IEEE Transactions on Wireless Communications, vol. 7, no. 12, pp. 5450 - 5460, December 2008.

[7] S. Tan, D. Z. J. Zhang, and J. R. Zeidler, "Distributed opportunistic scheduling for ad-hoc communications under delay constraints," in Proceedings of IEEE INFOCOM, San Diego, CA, March 2010.

[8] A. Banchs, A. Garcia-Saavedra, P. Serrano, and J. Widmer, "A game theoretic approach to distributed opportunistic scheduling." [Online]. Available: http://arxiv.org/abs/1107.4452

[9] F. Kelly, "Charging and rate control for elastic traffic," European Transactions on Telecommunications, vol. 8, pp. 33-37, 1997.

[10] B. Sadhegi, V. Kanodia, A. Sabharwal, and E. Knightly, "Opportunistic media access for multirate ad hoc networks," in Proceedings of ACM MOBICOM, Atlanta, GA, September 2002.

[11] P. Gupta, Y. Sankarasubramaniam, and A. Stolyar, "Random-access scheduling with service differentiation in wireless networks," in Proceedings of IEEE INFOCOM, Miami, FL, March 2005.

[12] B. Kristiansson and B. Lennartson, "Robust Tuning of PI and PID Controllers," IEEE Control Systems Magazine, vol. 26, no. 1, pp. 55-69, February 2006.

[13] A. K. Palit and D. Popovic, Computational Intelligence in Time Series Forecasting: Theory and Engineering Applications. Springer-Verlag New York, Inc., 2005.

[14] G. F. Franklin, J. D. Powell, and M. L. Workman, Digital Control of Dynamic Systems, 2nd ed. Addison-Wesley, 1990.

[15] G. Boggia, P. Camarda, L. A. Grieco, and S. Mascolo, "Feedbackbased control for providing real-time services with the 802.11e mac," IEEE/ACM Transactions on Networking, vol. 15, no. 2, April 2007.

[16] C. V. Hollot, V. Misra, D. Towsley, and W. B. Gong, "A Control Theoretic Analysis of RED," in Proceedings of IEEE INFOCOM, Anchorage, Alaska, April 2001.

[17] K. Aström and B. Wittenmark, Computer-controlled systems, theory and design, 2nd ed. Prentice Hall International Editions, 1990.

[18] W. C. Jakes, Microwave Mobile Communications. New York: John Wiley \& Sons Inc., 1975. 